\title{
Zum Ausdruck der Zukunft im Mittelhochdeutschen
}

\author{
Yvonne Luther
}

\begin{abstract}
Ausdrucksformen des Futurs sind für die historische Linguistik seit langem von Interesse. Jedoch liegt bisher für keine frühere Sprachstufe des Deutschen diesbezüglich eine umfassende Darstellung vor. Mit dem Bochumer Mittelhochdeutschkorpus steht ein strukturiertes und digital aufbereitetes Handschriften-Korpus zur Verfügung, das für eine umfassende Darstellung futurisch gebrauchter Formen ideale Voraussetzungen bietet. Die vorgestellte Untersuchung zukunftsbezogener Äußerungen im Mittelhochdeutschen stellt gegenüber den bisherigen semasiologischformorientierten Darstellungen einen Neuansatz dar: Ausgehend von der funktionalen Bestimmung von Äußerungen als ,zukunftsbezogen ' werden die entsprechenden ausdrucksseitigen sprachlichen Mittel erfasst. Auf diese Weise können die futurischen Ausdrucksmöglichkeiten des Mhd. in ihrer Komplexität analysiert und beschrieben werden. Im Folgenden wird ein Teil der Ergebnisse der Arbeit vorgestellt. Im Vordergrund steht dabei ein allgemeiner Überblick über den Gebrauch der wichtigsten Formen innerhalb des Untersuchungszeitraums.
\end{abstract}

\section{Untersuchungsgegenstand und Zielsetzung}

Der Status und die Verwendung des Futurs im Deutschen gehören zu den umstrittenen Bereichen der Tempusforschung ${ }^{1}$. Auch die Entstehung und Verbreitung der Form werden + Inf. hat noch keine allgemein anerkannte Erklärung gefunden und wird immer wieder kontrovers diskutiert².

Die bisherigen Arbeiten zu zukunftsbezogenen Äußerungen im Mittelhochdeutschen und anderen historischen Sprachstufen des Deutschen stellen meist eine oder mehrere bestimmte Formen (z.B. Modalverb + Inf., werden + Inf.) in den Mittelpunkt und werten diese hinsichtlich ihrer temporalen und modalen Verwendung aus oder sie konzentrieren sich auf Übersetzungen ${ }^{3}$.

Die hier vorgestellte Untersuchung basiert auf einem anderen Ansatz: Den Ausgangspunkt bildet die futurische Äußerungsbedeutung, der Untersuchungsgegenstand wird somit funktional bestimmt. Das Merkmal ,zukunftsbezogen“ wird einer Äußerung nur in Relation zum Äußerungszeitpunkt zugeordnet, d.h. Zukunftsbezug besteht, wenn das Zeitintervall, in dem das bezeichnete Ereignis stattfindet, gegenüber dem Äußerungszeitpunkt nachzeitig ist (vgl. auch Brons-Albert 1982: 13). Es ist dabei grundsätzlich nicht von Bedeutung, ob das Ereignis unmittelbar auf die Äußerung folgt oder erst in ferner Zukunft stattfindet. Auch eine Überlappung mit dem Sprechzeitpunkt ist nicht von vornherein ausgeschlossen, wenn sich das bezeichnete Ereignis in die Zukunft erstreckt. Modale

1 Im Mittelpunkt steht dabei vor allem das Futur I und seine temporale und modale Funktion, als Ausgangspunkt der Kontroverse gilt u.a. Saltveit (1960). In der Folge entstanden zahlreiche Arbeiten, die den Tempusstatus der Form infrage stellen (z.B. Vater 1975 und 1997).

2 Vgl. z.B. die Diskussion der wichtigsten diesbezüglichen Theorien bei Westvik (2000) sowie Abschnitt 4.4.2. im Folgenden.

3 Vgl. z.B. von Monsterberg-Münckenau (1886), Winkler (1913), Lussky (1924), Kleiner (1925), Lawson (1958), Walther (1980), Bogner (1994), Schmid (2000), Pfefferkorn (2005). 
oder andere Bedeutungsaspekte werden bei der Belegermittlung zunächst vernachlässigt, es ist somit unerheblich, ob ein Ereignis wahrscheinlich, sicher oder unwahrscheinlich eintritt; auch die Einstellung des Sprechers zur Aussage ist nicht von Interesse.

Auf diese Weise können alle Formen erfasst werden, die im Mittelhochdeutschen der Bezeichnung von Zukünftigem dienen. Aus der früheren Forschung ist bekannt, dass insbesondere das Präsens für futurische Äußerungen genutzt wird. Hinzu kommen seit dem Althochdeutschen verschiedene analytische Verbformen (Modalverbperiphrasen, werden + Part. Präs./Inf., vgl. Paul 2007: §S 4 zum Präsens und zu den analytischen Formen $§ \S S 12 \mathrm{ff}$.$) .$

\section{Korpus der Untersuchung}

Die Untersuchung wurde anhand einer Auswahl von Handschriften des Bochumer Mittelhochdeutschkorpus (BoMiKo) durchgeführt, da diese elektronisch verfügbar aufbereitet und somit für statistische Untersuchungen sehr gut geeignet sind. Sie ordnet sich damit in die aktuelle Forschung zum Mittelhochdeutschen ein, die auf handschrifliche Quellen zurückgreift ${ }^{4}$. Die Nutzung von Handschriften (i.d.R. in Form von Mikrofilmen oder Faksimiles) dient dazu, die früher verbreitete Editionspraxis der ,normalisierten` Textausgabe und die damit verbundenen Eingriffe in die Quellen zu überwinden (vgl. dazu auch Wegera 2000). Die Textauswahl hat das Ziel, die Gesamtheit der mhd. Überlieferung exemplarisch zu vertreten sowie deren Variabilität und diachrone Entwicklung zu berücksichtigen.

Das Bochumer Mittelhochdeutschkorpus ist hinsichtlich der Parameter ,Raum` und ,Zeit‘ strukturiert:

$\begin{array}{lll}\text { Zeitraum: } & \text { I } & 1050-1150 \\ & \text { II } & 1150-1200 \\ & \text { III } & 1200-1250 \\ & \text { IV } & 1250-1300 \\ & \text { V } & 1300-1350\end{array}$

Zeitraum $\quad$ I: $\quad 0$ obd.

II/III: 1 bair.; 2 alem.-bair.; 3 alem.; 4 wmd., 5 hess.-thür.

IV: 1 bair.; 2 alem.-bair.; 3 alem.; 4a mfrk; $4 \mathrm{~b}$ rhfrk-hess.; 5 omd.

V: 1 bair.; 2 alem.-bair.; 3 alem.; 4 a mfrk; 4 b rhfrk-hess.; 5 omd.; 6 ofrk. ${ }^{5}$

4 Das Korpus umfasst zurzeit 102 Texte mit ca. 1000000 Wortformen (vgl. dazu Klein 1991, Wegera 2000). Das seit 1997 durch die DFG geförderte Projekt „Mittelhochdeutsche Grammatik“ verfolgt das Ziel, eine neue wissenschaftlliche Grammatik des Mittelhochdeutschen zu erarbeiten (für Informationen zum Projekt vgl.

http://www.germanistik.uni-halle.de /arbeitsstellen/mittelhochdeutsche_grammatik/). Bereits erschienen sind Band III Wortbildung (Klein/Solms/Wegera 2009) sowie mehrere Einzeluntersuchungen (Herbers 2002, Leipold 2006, Waldenberger 2009).

50 obd. = oberdeutsch, 1 bair. = bairisch, 2 alem.-bair. = alemannisch-bairisch (Übergangsraum), 3 alem. = aleman- 
Neben dieser diatopischen und diachronen Strukturierung werden auch die drei Darbietungsformen Vers, Prosa und Urkunde unterschieden.

Eine erste Orientierung zur Textauswahl für die Untersuchung zukunftsbezogener Äußerungen lieferte eine computergestützte Suche im gesamten BoMiKo. Durch die Erfassung der bekannten futurisch genutzten Formen des Mhd. (Präsens, Konstruktionen aus sol/wil/muoz im Präsens + Inf. sowie werden im Präsens + Part. Präs. oder Inf.) wurde angestrebt, eine Einschränkung der zu untersuchenden Hss. hinsichtlich des Untersuchungsgegenstandes zu erreichen, ohne dabei jedoch ausschließlich Werke auszuwählen, die hauptsächlich zukunftsbezogene Inhalte bieten ${ }^{6}$. Somit sollte sichergestellt werden, dass der normale, d.h. auch nur gelegentliche Gebrauch futurischer Formen untersucht wird. Die Konzentration auf bereits inhaltlich auf futurische Sachverhalte geprägte Texte liefert zwar mit Sicherheit eine Vielzahl von Belegen, kann aber auch zu einer Verzerrung des Ergebnisses führen, da bei einer engen Abfolge entsprechender Äußerungen verstärkt mit stilistischen Einflüssen zu rechnen ist. So ist auch im Gegenwartsdeutschen eine Reihung von werden + Inf. in aufeinanderfolgenden futurischen Äußerungen eher unüblich (vgl. Duden-Gr. 2005: §737).

Nach der grundlegenden Vorauswahl wurde ein Untersuchungskorpus zusammengestellt, das alle Sprachlandschaften sowie Zeiträume des BoMiKo einschließt. Der Schwerpunkt des Korpus liegt auf Prosawerken, die durch 20 Hss. vertreten sind, hinzu kommen zehn Verstexte. Die im BoMiKo integrierte Überlieferungsform Urkunde wurde nur stichprobenartig zum Vergleich ausgewertet, da hier von anderen inhaltlichen Voraussetzungen und Merkmalen auszugehen ist ${ }^{7}$.

\section{Belegermittlung}

Zunächst wurde ermittelt, welche Verbalformen in den ausgewählten Texten in futurischen Äußerungen auftreten. Diese Bestimmung orientiert sich vor allem an kontextuellen und inhaltlichen Kriterien (z.B. Vorhandensein von Zeitangaben, Prophezeiungen, Verweis auf folgende Handlungen usw.; vgl. die folgenden Beispiele). Es wurde angestrebt, nur solche Belege zu wählen, die deutlich als zukunftsbezogen zu interpretieren sind (zur Vorgehensweise vgl. auch Pfefferkorn 2005: 314ff.).

nisch, 4 wmd. = westmitteldeutsch, $4 \mathrm{a}$ mfrk. = mittelfränkisch, $4 \mathrm{~b}$ rhfrk.-hess. $=$ rheinfränkisch-hessisch, 5 omd. $=$ ostmitteldeutsch, 6 ofrk. = ostfränkisch.

6 Vgl. z.B. die Vorgehensweise von Schmid (2000), der ein Korpus von Versionen der Prophezeiung der Vorzeichen des Jüngsten Gerichts zugrunde legt.

7 Es ist z.B. anzunehmen, dass Urkunden eine große Anzahl von Modalverbkonstruktionen (z.B. mit sol(e)n) aufweisen, wobei jedoch eine vorwiegend modale Interpretation der Äußerungen naheliegt. So stellt Pfefferkorn (2005: 316) fest, dass futurisch gebrauchte (also primär temporale) Modalverbkonstruktionen in Urkunden und Rechtstexten nur selten auftreten. 
a) morgen frì gib ich dir rat, III-0-V_Parz, 815, $9^{8}$

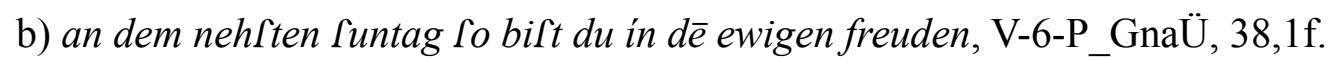

c) Er Ipach. ier werdent mich ain clain zît niht Tehende, IV-3-P_PrSch, 9v,10f.

Grundsätzlich erfolgte zunächst eine einzeltextbezogene Analyse, um individuelle Besonderheiten (z.B. Bevorzugung einer Form, bestimmte Vorkommenskontexte wie direkte Rede) zu ermitteln.

Dies erwies sich im Laufe der Untersuchung kontinuierlich als angemessene Vorgehensweise, da die einzelnen Hss. vielfach spezifische Gebrauchsweisen der futurischen Ausdrucksmöglichkeiten aufweisen.

Die betrachteten Belege sind i.d.R. Sätze oder Teilsätze, die ein finites Verb enthalten. Zusammengehörige Hauptsätze und Nebensätze werden getrennt als Belege gewertet, sofern beide als futurisch beurteilt wurden. Die Trennung in Haupt- und Nebensätze erscheint sinnvoll, da aufgrund bisheriger Erkenntnisse ein unterschiedlicher Gebrauch der Ausdrucksmöglichkeiten zu erwarten ist. Bereits Wilmanns stellt fest, dass der Gebrauch des Futur im „bedingenden Nebensatz“ (1906: §101) sowie in „Forderungs- und Absichtssätzen“ (ebd.) kaum oder nicht möglich ist. Damit einher geht die Beobachtung, dass Futurformen ,universaltypologisch selten in Sätzen mit temporalen oder konditionalen Konjunktionssätzen, die Zukunftsreferenz haben“ (Abraham 1989: 350) auftreten.

4. Entwicklung der primär genutzten Formen im Untersuchungszeitraum

\subsection{Allgemeiner Überblick}

Zum Ausdruck des Futurs wird im untersuchten korpus vorrangig das Präsens verwendet, daneben treten analytische Verbformen als sogenannte Futurumschreibungen auf. In beiden Fällen hängt die zukunftsbezogene Interpretation der entsprechenden Äußerungen von verschiedenen Faktoren, wie z.B. dem Vorliegen temporaler Markierungen (z.B. Adverbien, vgl. Paul 2007: §S 4), bestimmter Inhalte oder kontextueller Beziehungen ab (z.B. Wan Ii gebiret eine 「vn, V-5-P_MBeh, 55r,19Wiedergabe einer Prophezeiung, Ich wil ovch mit mir fivren. hundert miner man, III-0-V_Nib, 1307,1 - ein Sprecher verweist auf eine folgende Handlung seinerseits). Allgemein ist immer von

8 Die Siglen nehmen die Nummerierung der Zeiträume und Sprachlandschaften auf und enthalten eine Kennzeichnung der Textart (V für Verstext, P für Prosatext, U für Urkunde), hinzu kommt ein Kürzel des Texttitels (z.B. Parz für ,Parzival ${ }^{`}$ ). Zur Identifikation der hier zitierten Hss. s. Anhang. 
einem komplexen Zusammenwirken mehrerer Parameter auszugehen, das den Zeitbezug der jeweiligen Äußerungen bzw. der verwendeten Formen beeinflusst.

Die Futurumschreibungen des Mhd. sind in erster Linie die Modalverbperiphrasen mit sol(e)n, wellen, müezen im Präsens und dem Infinitiv des Vollverbs. Grundsätzlich ist anzunehmen, dass der modale Bedeutungsaspekt dabei nicht völlig zurücktritt; typisch ist vielmehr ein Schwanken zwischen vorwiegend modal-gegenwartsbezogener bzw. vorwiegend temporal-futurischer Funktion, das auch innerhalb desselben Textes auftreten kann (vgl. Paul 2007: §S 12) ${ }^{9}$. Offen bleibt dabei, ob hier bereits eine Bedeutungsvariante ,Zukunft‘ für die entsprechenden Konstruktionen anzunehmen ist oder ob lediglich eine Implikatur vorliegt (vgl. Welke 2005: 396). Auch der temporal-futurische Gebrauch schließt das Vorhandensein der modalen Bedeutungskomponente nicht generell aus, in vielen Fällen ist eine eindeutige Zuordnung nicht möglich (vgl. z.B. die Modalverbkonstruktionen mit muoz und sol im Folgenden: iwer minne ich haben wolte. mag ich der niht erwerben. ᄃo mi̊z ein Iowerz Iterben. Iich Cchiere an mír rezeigen. ir weltet iwer eigen. ob ich vriheit îe gewan. ir Ivlt mich doch fvr eigen han. daz dvnchet mich iwer ledech reht, III-0-V_Parz, 523,22ff.).

Die Konstruktion werden mit Part. Präs. kann ebenfalls als Futurumschreibung verwendet werden. Sie bezeichnet zunächst den Eintritt eines Zustandes oder Vorganges. Der Schwerpunkt liegt dabei auf dem neuen Zustand/Vorgang, wodurch die Aktionsartbedeutung von der temporalen (futurischen) Funktion überlagert wird (vgl. Schrodt 2004: §S 5, 1.c.). Die nhd. Futurform werden + Inf. tritt im Mhd. sehr selten und spät auf. Der Beginn ihrer Ausbreitung wird im Allgemeinen in der zweiten Hälfte des 14. Jh.s. angesetzt (vgl. Paul 2007: §S 13, A4) ${ }^{10}$. Der Wendepunkt für die vollständige Ablösung der Modalverbperiphrasen durch das werden-Futur liegt im16. Jh. ${ }^{11}$. Die Modalverbformen bleiben jedoch bis ins 18. Jahrhundert im futurischen Gebrauch erhalten.

\subsection{Präsens}

Bezüglich des Präsens wird auch für die Gegenwartssprache allgemein angenommen, dass es „die wenigsten kontextuellen Distributionsbeschränkungen“ (Thieroff 1992: 89) aufweist und als unmarkiertes Tempus die meisten Anwendungsmöglichkeiten besitzt. Aufgrunddessen ist es nicht mög-

9 Bereits im Ahd. treten Umschreibungen mit sculan, mugan und selten wellen auf, auch hier wird gelegentlich thanne gebraucht, um den futurischen Zeitbezug zu verdeutlichen. Im Einzelfall ist meist fraglich, ob von einer futurischen oder einer primär modalen Fügung auszugehen ist (vgl. Schrodt 2004: §S 124).

10 Laut Schrodt (2004: §S 124) sind Behauptungen, die die Futurumschreibung mit werden und Inf. bereits im Tatian annehmen, als unberechtigt anzusehen. In ahd. Übersetzungstexten wird das lat. Futur i.d.R. durch das Präsens wiedergegeben. Bei Otfried wird der Zukunftsbezug oftmals durch Adverbien (z.B. thanne, noh, furdir) verdeutlicht.

11 Vgl. Ebert/Reichmann/Solms/Wegera (1993: §S 167), Kotin (2003: 158f.), Bogner (1989: 74ff.), Szatzker (2002: 46). 
lich, dieses Tempus über eine Relation von Sprechzeitpunkt zu Ereignis zu bestimmen. Es wird zudem immer dann verwendet, wenn eine zeitliche Orientierung unerheblich ist. Vielfach wird das Präsens daher mit Bezeichnungen wie ,Nicht-Vergangenheitstempus‘ (vgl. Thieroff 1994: 121) oder ,Tempus der Nicht-Abgeschlossenheit‘ (vgl. Gelhaus 1969: 17) in Verbindung gebracht. In der Umgangssprache ist das Präsens normalerweise die meistgenutzte Ausdrucksmöglichkeit in futurischen Äußerungen, in der Schriftsprache wird das Futur I meist deutlich häufiger gebraucht ${ }^{12}$. Zudem ist anzunehmen, dass nicht nur Kontext und Situation bei der Disambiguierung des Präsens wirksam sind, sondern auch die Semantik bzw. Aspektualität des finiten Verbs, die eine grundlegende inhärente Temporalität vorgibt.

Bereits Behaghel (1924: 112) schreibt dem Präsens eines durativen Verbs Gegenwartsbezug, dem eines perfektiven Verbs jedoch im Allgemeinen Zukunftsbezug zu. Welke (2005: 424) schränkt dies für die Gegenwartssprache dahingehend ein, dass nur achievement-Verben (z.B. kommen, erreichen, fallen) die Default-Bedeutung Zukunft haben. Es handelt sich hier um eine Kategorie der Verbbedeutung, d.h. der Zeitbezug wird nicht allein durch die Tempuskategorie Präsens manifestiert (zum Einfluss der Aspektualität auf die Tempusbedeutung vgl. auch Saltveit 1962: 10f., Leiss 1992: 191ff., Žuikin 1975).

Im untersuchten Korpus ist das futurische Präsens im Allgemeinen die vorrangig genutzte Ausdrucksform für zukünftige Sachverhalte. Die futurische Referenz kann dabei durch Zeitangaben unterstützt werden (z.B. ez chvmt noch an die Itunde. vil liht in churcer cit, III-0-V_Nib, 2401,3), jedoch ist in der Regel beim Präsens-pro-futuro kein gehäufter Gebrauch entsprechender sprachlicher Mittel zu beobachten.

Die diachrone Betrachtung des futurischen Präsens zeigt eine Abnahme seines relativen Anteils am Gesamt aller Futurformen: Die Hss. aus Zeitraum I bevorzugen das Präsens besonders deutlich als Ausdrucksform für Zukünftiges, es erscheint in ca. 70\% aller futurischen Belege. In Zeitraum II wird in knapp 63\% der futurischen Äußerungen das Präsens verwendet (z.B. ir uindit אprach der engel êin chint. mit tiochelinin gewindelotiz. ift geleit in eine grippe, II-2-P_Spec, 9r,5ff.). In allen späteren Zeiträumen weisen nur noch etwas weniger als die Hälfte aller zukunftsbezogenen Äußerungen das einfache Präsens auf. Diese Veränderung vollzieht sich zugunsten der Gruppe der Modalverbperiphrasen, die dementsprechend höhere Anteile am Gesamt der futurischen Belege stellen. Bei Betrachtung aller Einzelformen bleibt das Präsens jedoch meist die vorherrschende Ausdrucksmöglichkeit in futurischen Äußerungen, nur wenige Hss. bevorzugen andere Formen deutlich (z.B. $w i l+$ Inf. in V-6-P_GnaÜ, sol + Inf. in V-4a-P_Taul).

Vor allem ab Zeitraum III lässt sich bezüglich des Formengebrauchs ein Unterschied zwischen Haupt- und Nebensätzen beobachten, letztere weisen im Allgemeinen höhere Anteile des futurischen Präsens auf (durchschnittlich 60\%), die Varianz zwischen den einzelnen Hss. ist jedoch z.T.

12 Vgl. Duden-Gr. (2005: §737), Vater (1997), Brons-Albert (1982: 102), Žuikin (1975). 
sehr stark.

Auch in der Gegenwartssprache ist werden + Inf. in Nebensätzen meist unüblich, selbst wenn sie sich auf Zukünftiges beziehen (eine Tendenz zur Neutralisierung von expliziten Futurformen in bestimmten Nebensätzen ist auch übereinzelsprachlich zu beobachten, vgl. z.B. Ultan 1988: 96ff.). Vater (1975: 100ff.) nennt in diesem Zusammenhang Temporalsätze, damit-Sätze, Wunschsätze und Befehlssätze, z.B. Wenn die Uhr sechs schlägt, zünden wir die Kerzen am Weihnachtsbaum an statt *Wenn die Uhr sechs schlagen wird, [...]. Er erklärt die Bevorzugung des Präsens damit, dass die durch werden + Inf. ausgedrückte Modalität mit der intendierten Bedeutung der Sätze, z.B. Erfüllung eines Wunsches oder Befehls, nicht kompatibel sei. Dies ist nicht selbstverständlich, so muss auch Vater (1975: 138, Anm. 36) seine Schlussfolgerung dahingehend abschwächen, dass nur „bestimmte Modalitäten“ ausgeschlossen seien. Ein Satz wie Peter arbeitet Tag und Nacht, damit er das Examen bestehen kann erscheint keineswegs ungewöhnlich, das Gleiche trifft für durch den Konjunktiv ausgedrückte Modalität zu (z.B. Der Kommandant befiehlt, dass man die Gefangenen vorführe, vgl. Welke 2005: 439). Als mögliche Erklärung für den Verzicht auf das Futur kann Redundanz angenommen werden, denn bereits aus dem übergeordneten Verb oder der Konjunktion geht hervor, ,dass das im Nebensatz ausgedrückte Ereignis zukünftig sein würde - falls sich das implizierte Ereignis realisiert“ (Welke 2005: 437). Da Redundanz allein nicht für ein „solch zwingendes Ergebnis“ (ebd.) spricht, erklärt Welke den Präsensgebrauch aus dessen Bedeutung der ,Zeitungebundenheit‘. Das Präsens ermöglicht eine Assertion außerhalb der Zeit wie etwa Zwei mal zwei ist vier. Es ermöglicht zudem, die Assertion des Nebensatzes ,überhaupt zu übergehen" (Welke 2005: 438), z.B. bei Emil wünscht, dass Erna ihn besucht. Das wirkliche Eintreffen des benannten Sachverhalts wird nicht behauptet. Da durch das Präsens diese Mitbehauptung umgangen werden kann, erscheint es hier als das geeignete Tempus.

\subsection{Modalverbperiphrasen}

Die mittelhochdeutschen Modalverben wellen, sol(e)n, müezen, mügen, kunnen, durfen tragen im weitesten Sinne eine voluntative oder deontische Grundbedeutung. Diese gibt dem entsprechenden Modalverb „einen aktionsartlichen Rahmen vor, der die Realisation der Prädikation als im Moment der Äußerung noch ausstehend bezeichnet“ (Fritz 1995: 51). Somit liegt die Grundvoraussetzung vor, dass sich futurische Bedeutung entwickeln kann bzw. futurischer Gebrauch möglich ist. Der tatsächliche Eintritt des antizipierten Sachverhalts ist möglich, jedoch ungewiss, es besteht eine Art Realisationsdruck bezogen auf eine sich in die Zukunft ausdehnende Verwirklichungszeit. Der ,auf den Eintritt eines Sachverhaltes hinweisende Charakter der Modalverben“ (ebd.) ist nicht auf die gewöhnlich in Futurperiphrasen erscheinenden Verben ( $\operatorname{sol}(e) n$, wellen, müezen) beschränkt, sondern ,wird allgemein als ein wichtiges semantisch konstitutives Merkmal der Verbgruppe systematisiert" (ebd.).

Im Verlauf der Grammatikalisierung einer Konstruktion Modalverb (im Präs.) + Inf. als Futurumschreibung tritt die jeweilige Grundbedeutung mehr und mehr in den Hintergrund bzw. geht verloren (vgl. Fritz 1995: 52). Es findet schließlich eine Verlagerung des semantischen Schwerpunkts auf das Ergebnis statt; nicht mehr die Art des Realisationsdrucks steht im Vordergrund, sondern die Proposition selbst (und ihre ausstehende Verwirklichung). Es entsteht somit ein zeitlicher Kontrast zwischen einem Bereich der Nicht-Verwirklichung und einem nachfolgenden der Verwirklichung. Die temporal vorausweisende Markierung der Konstruktion wandelt sich aktionsartbezo- 
gen von prospektiv zu terminativ, der Fokus wechselt vom Subjekt zur Gesamtaussage (vgl. auch Bybee 1985: 159).

Übereinzelsprachliche Untersuchungen (z.B. Bybee/Pagliuca 1987 und Bybee/Perkins/Pagliuca 1994) zeigen, dass modale Kategorien neben Fortbewegungsverben die häufigsten Ausgangselemente für Futurtempora darstellen (vgl. auch Ultan 1988). Der Ausdruck von Indefinitheit, der zu den grundlegenden Funktionen von Modalverbkonstruktionen gehört, bildet eine Voraussetzung für ihre Eignung als Ausgangsformen für Futurperiphrasen. Als weiterer wichtiger Faktor kann hier auch die meist relativ hohe Gebrauchshäufigkeit der Ausdrücke gelten.

Die futurische Verwendungsweise der Verben sol(e)n, wellen und müezen im Mhd. stellt keine konsequent grammatikalisierte Form dar, sondern ist eine „Zwischenstufe zwischen deontisch-modaler und epistemisch-modaler Bedeutung““ (Fritz 1995: 52).

Für andere germanische Sprachen, die Futurformen auf der Basis von Modalauxiliaren entwickelt haben, ist ebenfalls anzunehmen, dass die Funktion der ,reinen Voraussage' nicht kontextunabhängig realisiert werden kann (vgl. Abraham 1989: 365, Dahl 1985: 107f.). Aufgrund des parallelen Vorhandenseins der modalen Grundbedeutung bei diesen Futurformen nimmt Abraham an, dass ,ein Sog nach einem ,rein prädikativen “, amodalen Futur entsteht“ (ebd.), der durch aspektuelle oder andative/venitive Futurtypen abgedeckt wird.

Bybee/Pagliuca (1987: 122) gehen davon aus, dass futurisch gebrauchte Modalverbkonstruktionen zunächst ein willensfähiges und belebtes Agens verlangen, da die modalen Grundbedeutungen sich auf interne (z.B. Willen) oder externe, soziale Bedingungen (z.B. Verpflichtung) beziehen. Durch die Grammatikalisierung als Futurform werden diese Bedeutungsaspekte abgeschwächt und das Funktionsmerkmal ,Voraussage' tritt in den Vordergrund. Die Konstruktionen sind dann für alle Arten von Agenzien einsetzbar.

\subsubsection{Wil + Infinitiv}

Die Verbindung wil + Inf. gehört vor allem in den späteren Hss. (ab IV) zu den am stärksten verbreiteten futurischen Ausdrucksmöglichkeiten im Korpus. Ihr Anteil am Gesamt aller zukunftsbezogenen Äußerungen nimmt diachron betrachtet von I bis V von ca. 7\% auf 17\% zu.

Obwohl die Form allgemein verbreitet ist, unterscheidet sich die Gebrauchshäufigkeit in den einzelnen Hss. z.T. erheblich, so weist inZeitraum I Will einen Anteil von fast einem Fünftel futurischer Äußerungen mit wil + Inf. auf (z.B. İch uuíl uáran ze démo mírrebérge. unte ze démo uuîrôuchbúhele, 20v, 18ff.), während die Form in WNot bei Betrachtung aller Belege marginal ist (z.B. fore dero gotel chumfti unaret ir ungiloubig. unellet ir ouh noh ᄃo ؟in, 6ra, 4ff.). In Zeitraum II und III wird wil + Inf. insgesamt seltener verwendet als sol + Inf., das in beiden Zeiträumen in fast allen Hss. die bevorzugte Modalverbkonstruktion ist. Für die Mehrzahl der Hss. ab Zeitraum IV ist $w i l$ + Inf. die bevorzugte Modalverbkonstruktion. Insgesamt überwiegt wil gegenüber sol in Zeitraum IV leicht, in V erreichen beide Modalverben fast gleiche Anteile am Gesamt aller futurischen 
Äußerungen.

Insbesondere bei wil + Inf. ist eine direkte Korrelation zwischen gewählter Form und grammatischer Person und Ausdruckswert bzw. Funktion der Äußerung anzunehmen. Vorherrschend ist in der Regel die 1. Ps. Sg., durchschnittlich die Hälfte aller futurischen Belege mit wil sind hier einzuordnen. Typischerweise handelt es sich dabei um Äußerungen in direkter Rede, die folgende Handlungen ankündigen, z.B. ich wil an iv ertwingen. Swaz ir mugt han, III-0-V_Nib, 110,3, ich wil einen gefelle zi̊ mir nemē, V-3-P_NikP, 51rb,17f. In den Zeiträumen IV und V wird wil + Inf. auch häufiger in der 3. Sg. gebraucht (z.B. Er wil ím lonen nach dem allerínrlichoftem zůker des wille, V-3-P_NikP, 56va,4ff.), dennoch bleibt die 1. Sg. Schwerpunkt des Formengebrauchs. Hier lässt sich ein deutlicher Bezug zur modalen Grundbedeutung des Hilfsverbs beobachten, der darauf verweist, dass kein Übergang zu einem, reinen'Futur stattfindet.

\subsubsection{Sol + Infinitiv}

Sol + Inf. tritt in den frühen Hss. insgesamt noch seltener auf als wil + Inf., in den späteren Zeiträumen, besonders ab IV, erreichen beide Modalverbkonstruktionen ähnliche Anteile am Gesamt aller futurischen Äußerungen (bei sol + Inf. ca. 13\% in IV, ca. 17\% in V). Die Gebrauchshäufigkeit von $s o l+$ Inf. in den einzelnen Hss. ist z.T. stark verschieden, eine Bevorzugung der Form zeigen z.B. III-5-P_PrMK, IV-4a-P_Lilie, V-4a-P_Taul und V-5-P_MBeh. Für diese Hss. ist im Allgemeinen eine niedrigere Verwendungsfrequenz des futurischen wil + Inf. festzustellen.

Eine Schwerpunktbildung bezüglich der grammatischen Person ist bei sol + Inf. nicht in dem Maße wie bei wil + Inf. zu beobachten, nur in II erscheint die Form vorwiegend in der 2. Sg. und 2. Pl. (z.B. Dine chonin Caraý. die Coltu niemer heizzen Saraý. daz ift geantvrift. mîne frovwen, II-2P_Spec, 13r,2ff. - hier ist durch den Gebrauch des Modalverbs eine auffordernde Interpretation naheliegend, trotzdem bezieht sich die Äußerung deutlich auf etwas Zukünftiges). Ab III wird sol + Inf. überwiegend in der 3. Sg. verwendet, in V stehen nahezu 55\% der Belege in der 3. Sg. (z.B. He Cal dich hi behalden. inde Cal dir dar na lonen, IV-4a-P_Lilie, 5,32f.; Her Cal uch toufè ì dem heilig $\bar{e}$ geifte $v \bar{n}$ i fure, V-5-P_MBeh, 57r,13).

Sol + Inf. wird vorwiegend in Voraussagen verwendet, der Bezug zur modalen Grundbedeutung scheint den Formengebrauch weniger stark zu beeinflussen als bei wil + Inf. Tritt die Konstruktion in der 2. Ps. Sg./P1. auf, ist oftmals ein auffordernder Charakter der Äußerung implizit, vgl. z.B. daz

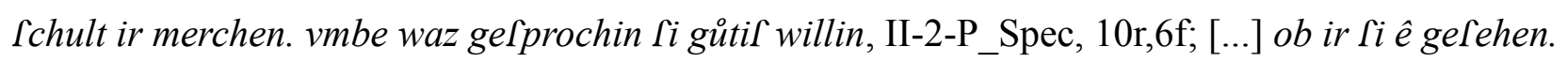
habt in vremden landen. des $\int v l t$ ir hagene mir veriehen, III-0-V_Nib, 83,3f. Andererseits kann 
auch in der 2. Ps. der Ausdruck einer Aufforderung deutlich hinter die Voraussage zurücktreten, vgl. z.B. du folt viber drizig tage komen in die Stat der ewigē vro̊den, V-3-P_NikP, 44ra,6ff., Noch hute Caltu mit mir fin ì dem paradýle, V-5-P_MBeh, 233r,1f.

\subsubsection{Muoz + Infinitiv}

Innerhalb der futurisch verwendeten Modalverbkonstruktionen ist muоz + Inf. im Korpus wenig verbreitet. Mit Ausnahme von Zeitraum II steht muоz bezüglich der Gebrauchshäufigkeit immer an dritter Stelle hinter wil und sol. Im Gegensatz zu wil und sol + Inf. ist hier diachron zudem eher eine abnehmende Verwendungshäufigkeit zu beobachten, in Zeitraum V stellt muoz + Inf. nur noch 1,5\% aller futurischen Belege. In der Konstruktion mit тиоz erscheint die modale Grundbedeutung des Hilfsverbs meist stark präsent, vgl. z.B. wir mvzin niderriten. in derHvnin lant, III-0-V_Nib, 1326,3, Ich mi̊z an dirre haíligen Itat Iterben, V-1-V_MMag, 484. Es ist somit vermutlich weniger als wil und sol geeignet, auch neutrale oder zumindest tendenziell neutrale Zukunftsbedeutung zu vermitteln.

\subsection{Konstruktionen mit werden}

Das Verb werden tritt als Auxiliar in mehreren Konstruktionen auf, die an der Umgestaltung der Beziehungen im System von Tempus, Modus und Aspekt im Laufe der Sprachentwicklung zum Nhd. hin zentral beteiligt sind. Grundsätzlich kann davon ausgegangen werden, dass neben dem Vollverb werden mehrere Auxiliarvarianten existieren, wobei das Hilfsverb zunächst auf den semantischen Merkmalen des Vollverbs aufbaut und diese dann weiterentwickelt (vgl. Fritz 1997: 82).

\footnotetext{
Noch im Ahd. (wie auch im Gotischen, vgl. Eroms 1992: 231) kann ein aspektuelles Paar werdan - wesan gebildet werden, wobei werdan die perfektive Funktion übernimmt und den Eintritt in einen Zustand enkodiert, wesan drückt das Verweilen in einem Zustand aus und bildet die imperfektive Ausdrucksvariante. Im Vergleich mit den futurisch gebrauchten Modalverbkonstruktionen ist festzuhalten, dass werden und die entsprechenden Modalverben im Ahd. unterschiedliche Grundbedeutungen zeigen: während die Modalverben über eine additive Semantik verfügen, drückt werden Unteilbarkeit aus. Es lässt sich somit schließen, dass nicht wie bei den Modalverben die additiv-prospektive Eigenschaft zur Futurinterpretation von Konstruktionen mit werden führte, sondern die perfektive Qualität des Auxiliars.

Vom Ahd. zum Mhd. beginnt sich die starke Grenzbezogenheit von werden zu verringern, an der Wende zum Frnhd. hat es sich den formalen und inhaltlichen Mustern der Modalverben weitgehend angeschlossen. Der Zukunftsbezug von werden + Inf. ist an das terminative Schema der Modalverben angelehnt (vgl. Fritz 1997: 88). Auch die beteiligte Infinitivform ist geeignet, zur Futurverwendung beizutragen, da sie nicht wie das Partizip Präteritum ,Abgeschlossenheit‘ oder das Partizip Präsens, Verlauf" signalisiert (vgl. Bybee/Pagliuca 1987: 111). Im Hochdeutschen verdrängt werden + Inf. die zukunftsbezogenen Modalverbkonstruktionen, wobei hier ein Zusammenhang mit der Ausdifferenzierung der epistemischen Bedeutungen der Modalverben anzunehmen ist.
} 


\title{
4.4.1. Werden + Partizip Präsens
}

Die im Ahd. selten, im Mhd. häufiger vertretene Form werden + Part. Präs. wurde ursprünglich zur Kennzeichnung von Ingressivität ${ }^{13}$ verwendet: „,ein Zustand/Vorgang wird beendet, die Handlung geht in einen neuen Zustand über“ (Schrodt 2004: §5, c).

\begin{abstract}
Der progressive Ausdruckswert der Verbindung ist dabei weniger an das finite Auxiliar geknüpft als an das Partizip Präsens. Während letzteres im Nhd. in erster Linie als Adjektiv erscheint, besitzt es im Ahd. noch starke Verlaufsbedeutung (zum Verlaufscharakter von werden + Part. Präs. vgl. Welke 2005: 378ff.). Somit trägt im Ahd. das Partizip Präs. die additive Komponente und werden drückt den punktuellen Zustandswechsel aus. Welke (2005: 390) geht davon aus, dass die Konstruktion wahrscheinlich keine Entwicklung zu einem Futur durchlaufen hat, sondern ihre Verlaufslesart beibehielt. Der futurische Gebrauch erklärt sich somit daraus, dass das Präsens dieser Form - wie jedes Präsens - in zukunftsbezogenen Äußerungen gebraucht werden konnte (zum möglichen Unterschied zw. perfektiven und imperfektiven Verben im Part. Präs. vgl. Welke 2005: 391).
\end{abstract}

Das zukunftsbezogene werden + Part. Präs. ist im untersuchten Korpus vor allem bis Zeitraum III nur vereinzelt vorhanden. Die Form tritt in wenigen Hss. und meist in Einzelbelegen auf (z.B. Cam

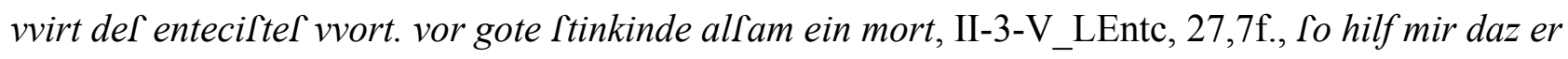
lebinde werde, III-5-P_PrMK, 3v,30f.).

In Zeitraum IV und V ist werden + Part. Präs. insgesamt deutlich häufiger belegt, es ist jedoch weiterhin auf einzelne Hss. beschränkt (z.B. IV-3-P_PrSch, V-2-P_Baum, V-3-P_NikP) und tritt nicht als allgemein verbreitete futurische Ausdrucksform in Erscheinung. Die Verwendungshäufigkeit variiert bei den verschiedenen Hss. von singulärem oder marginalem Auftreten (IV-5-P_AthP, V-6-P_GnaÜ) bis hin zu einem Anteil von über einem Zehntel aller futurischen Belege (in IV-3P_PrSch, z.B. Er Ipach. ier werdent mich aín clain zît niht 「ehende, 9v,10f. - Übersetzung für Modicum et iam non videbitis me; wan fo wirt fich íuwer herze fröwende, 10v,14f. - Übersetzung für et gaudebit cor vestrum). Eine Weiterentwicklung der Form in Richtung eines Futurtempus oder eine zunehmende Verbreitung kann aus der diachronen Gegenüberstellung nicht abgeleitet werden.

\subsubsection{Werden + Infinitiv}

Die Herausbildung und Verbreitung der Form werden + Infinitiv ist ein Schwerpunktthema der Tempusforschung, sie gilt jedoch noch immer als umstritten. Sicher nachzuweisen ist die Fügung seit dem 13. Jahrhundert (vgl. z.B. Walther 1982: 597). Wiederholt wurde vor allem die Annahme einer frühen Entstehung von werden + Inf. zurückgewiesen. Westvik hält dazu fest, dass die äußerst

13 Die Begriffe ,ingressiv` und, inchoativ` werden in der Forschungsliteratur vielfach synonym verwendet. Gerade für die Konstruktion werden + Part. Präs. erscheinen beide Bezeichnungen, so z.B. ingressiv in der Ahd. Gr. (Schrodt 2004: $\$$, c) und inchoativ in der Mhd. Gr. (Paul 2007: §28, 4.2.2. und §13, 5: ingressiv oder inchoativ). Aufgrund des an der Konstruktion beteiligten Partizips Präsens ist zunächst die Annahme einer Zustandscharakterisierung naheliegend (ähnlich wie bei einem Adjektiv). 
geringe Zahl an Beispielen aus der Zeit vom 8./9. bis 13. Jh. unverständlich bleibt, wenn von einer vor- oder frühahd. Entstehung ausgegangen wird - zudem seien viele dieser Belege ,philologisch anfechtbar“(Westvik 2000: 240).

In der Forschungsliteratur lassen sich mindestens sechs verschiedene Erklärungsansätze zu wer-

$d e n+$ Inf. unterscheiden, die z.T. in verschiedenen Varianten auftreten: Abschleifungstheorie, Analogietheorie, Kontaminationstheorie, Autonomietheorie, Konfusionstheorie und Interferenztheorie (vgl. Westvik 2000, Kotin 2003: 155ff.).

Die ältere Forschung betrachtete die Konstruktion in erster Linie als lautlich verursachte Erscheinung, d.h. als Resultat eines Abschleifungsprozesses des Part. Präs. in der Verbindung mit werden (vgl. z.B. Bech 1901, Weinhold, Mhd.Gr.: $\$ 435$ und die restringierte Theorie bei Behaghel 1924: 262f.). Demzufolge habe die Endung des Part. Präs. -ende sich zu -en abgeschliffen und das Part. Präs. wäre mit dem Inf. zusammengefallen, es folgte die Umdeutung des Part. Präs. zum Infinitiv (Bsp. für die phonologische Entwicklung z.B. bei Bech 1901). Dass die Abschleifung des Part. Präs. vor allem im niederdeutschen Sprachgebiet nachgewiesen ist, scheint mit dem Auftreten der ältesten Belege für werden + Inf. im Obd. unvereinbar.

Die in der Mhd.Gr. (Paul 2007: §S 13, A.4) erwähnte These von einer Vermischung des Part. Präs. mit dem (zunächst) flektierten Infinitiv orientiert sich an Kleiner (1925). Die Konfusionstheorie stützt sich darauf, dass die Verbindung werden + Part. Präs. im Mhd. Zukunftsbedeutung entwickelte sowie darauf, dass der flektierte Inf. dem Part. Präs. weitgehend formal gleicht. Das Vorkommen der flexionslosen Infinitivform anstelle der flektierten führte zu der Annahme, dass dieser Infinitiv auch in die werden-Fügung eingedrungen sei.

Von einer Analogiebildung geht Wilmanns (1906: 177) aus, demnach habe sich die Verbindung nach dem Vorbild von ahd. biginnan und gistantan entwickelt, die mit dem Infinitiv verbunden werden. Der lautliche Verfall der Partizipialendung und die Vermischung mit dem Inf. werden zusätzlich als einflussreich für die Verbreitung der Form angesehen.

Saltveits (1962) Autonomietheorie geht von einer separaten Entstehung der Infinitiv-Verbindung aus und nimmt für diese ursprünglich modale Bedeutung an. Er kommt zu dem Schluss, dass es keine direkte diachrone Abfolge von werden + Part. Präs. und werden + Inf. gab und sieht werden + Adj./Subst. als Vorläufer der Futurform. Da auch für werden + Part. Präs. zumindest z.T. futurischer Gebrauch anzunehmen ist, kann hier ein zusätzlicher Einfluss vorliegen. Beide Fügungen konnten vermutlich im Präsens unabhängig voneinander Zukunft ausdrücken (vgl. Saltveit 1962: 248 und 253). Er stützt seine Erklärung auch auf das Altnordische, das die Struktur verpa + Infinitiv mit modaler Bedeutung besitzt sowie die norwegische Dialektform bli + Inf., die als Futur fungiert (dabei ist bli im Norwegischen Ersatz für älteres verpa). Hinzu kommt der „Hinweis darauf, daß werden etymologisch gesehen einem Bewegungsverb nahekomme“ (Westvik 2000: 239), was dazu führe, dass der beteiligte Infinitiv adverbial gedeutet werden müsse (vgl. Saltveit 1957: 227). Infolge dieser Überlegungen nimmt Saltveit an, dass werden + Inf. eine normale syntaktische Struktur sei, die sich wie die anord. Form erklären lasse.

Leiss (1985 und 1992: 197ff.) geht davon aus, dass die Entwicklung des analytischen Futurs mit werden auf deutsch-tschechische Sprachkontakte zurückzuführen ist und im Ostmitteldeutschen ihren Ausgangspunkt hat (Interferenztheorie). Sie stützt sich auf die Beobachtung Kleiners (1925), dass sich die Form im Alemannischen von Osten nach Westen ausbreitet, und beruft sich außerdem auf die Untersuchungen von Walther (1982) und Schieb (1981), die ihre These zu unterstützen scheinen. Leiss' Argumentation ist jedoch kritisch zu betrachten, da Kleiner die Ausbreitung von werden + Inf. im Alemannischen ungefähr zwischen 1375-1450 ansetzt, die Arbeiten von Schieb und Walther sich jedoch auf die Zeiträume 1470-1530 bzw. 1450-1550 beziehen. Daraus ergibt sich die Frage, ob die zeitlich später einsetzenden Korpora überhaupt Aussagen über die Herkunft und Ausbreitung der Fügung erlauben.

Schmid (2000) identifiziert die Fügung als ursprünglich oberdeutsch. Für seine Untersuchung nutzte er ein Korpus von über 100 Versionen der Prophezeiung der Vorzeichen des Jüngsten Gerichts vom 12. bis zum 17. Jh. (geographisch vom Mittelniederländischen/Mittelniederdeutschen bis zum Obd.) und erhielt somit geeignetes Quellenmaterial, das sich inhaltlich klar auf Zukünftiges bezieht. Bis ins 14. Jh. tritt werden + Inf. nur in wenigen Texten auf, erst im 15. Jh. erscheint es häufiger, in obd. Texten bereits bei 16 von 37 Texten als Hauptform des Futurausdrucks. Im Md. tritt die Fügung deutlich seltener auf und bleibt Nebenform (vgl. Schmid 2000: 13). Auf dieser Grundlage folgert Schmid, dass werden + Inf. ursprünglich im Obd. zu finden sei. Westvik weist darauf hin, dass ein ,schiefe geographische Verteilung der Texte besonders im 14. und 15. Jh.“ (2000: 242) vorliege. Auch wenn anzunehmen ist, dass die von Schmid für das Obd. gewonnenen Ergebnisse aufschlussreich sind, ist durch die Untersuchung nicht der Nachweis erbracht worden, dass dort das Ursprungsgebiet von werden + Inf. zu finden ist. Die Kontaminationstheorie behandelt semantisch äquivalente Ausdrücke wie $x$ wird, $x$ soll werden/kommen und $x$ wird werden/kommen. 
Aufgrund der diesbezüglich ermittelten Belege nimmt Schmid an, dass der syntaktische Typ $x$ wird werden auf Kontamination von $x$ wird und $x$ soll werden beruhen könnte. Als weitere Basis können Ausdrücke mit Prädikatsnomen dienen: $a$ wird $b$ und $a$ soll $b$ werden wird $\mathrm{zu} a$ wird $b$ werden (vgl. Schmid 2000: 13ff.). Die Hypothese hat gegenüber anderen Theorien den Vorteil, dass sie unabhängig von Zeit und Ort sowie anderen sprachlichen Entwicklungen ist. So kann Schmid die Form wirdit bezzera sîn bei Otfried als Kontamination von wirdit bezzira und skal bezzira sîn erklären (vgl. 16f.). Es ist allerdings davon auszugehen, dass die angenommenen Kontaminationen (wird werden, sein, kommen) zunächst spontan aufgetreten sein müssten, um dann wiederholt zu erscheinen und als komplexe Futurformen analysiert zu werden: „Erst danach kann man weitere Verbindungen wie wird sagen, wird sterben, wird helfen, usw. gebildet haben, die nicht selbst als Kontaminationen aufgefaßt werden können“ (Westvik 2000: 257). Ein Beweis wäre erbracht, wenn zumindest eine Zeitlang nur reine Kontaminationsprodukte nachweisbar wären was allerdings nicht der Fall ist. Somit wäre es durchaus möglich, alternative Kontaminationstheorien zu formulieren, z.B. werden + Part. Präs. und sollen + Inf. führen zu werden + Inf. (wirt komende + sol komen = wird komen, vgl. Westvik 2000: 258).

Als „Archetyp sowohl der Zukunftsbedeutung als auch der epistemischen Bedeutung“ (Welke 2005: 377) des gegenwartssprachlichen Futurs kann die prädikative Konstruktion aus werden und Adjektiv/Substantiv (Er wird reich/Lehrer) angenommmen werden (vgl. auch Kotin 2003: 155ff. und Saltveit 1962). Strittig bleibt jedoch, wie es zu den vorliegenden Funktionen der Form kam. Bezüglich der beiden gegenwartssprachlichen Bedeutungsvarianten des Futur I - temporal-futurisch oder modal-gegenwartsbezogen - wird allgemein davon ausgegangen, dass die Zukunftsbedeutung die ursprüngliche ist - dies wird durch synchrone Rekonstruktion sowie durch diachrone Gesichtspunkte bestätigt (vgl. z.B. Saltveit 1962: 23ff., Kotin 2003: 168, Szatzker 2002). Auch die quantitativen Verhältnisse in gegenwartssprachlichen Texten sprechen für die Priorität der Zukunftsbedeutung, da der Anteil des gegenwartsbezogenen epistemischen werden + Inf. nur sehr gering ausfällt ${ }^{14}$.

Im untersuchten Korpus ist werden + Inf. als Futurform insgesamt eine Randerscheinung, die sich auch in Hss. aus V nur selten belegen lässt, z.B. Selic fit ir di nu weinet. Wan ir werdet lachin, V-5-P_MBeh, 146r,23f., du Cihft mich ietzund neur als durch eínē flímē. her nach als du mich wirdeft Tehen durch den Ipigel miner gotheit, V-6-P_GnaÜ, 87,8ff. Insgesamt stellt die Form in V weniger als $1 \%$ aller futurischen Belege. In den Hss., in denen werden + Inf. auftritt (MMag, MBeh, GnaÜ), gehört es gewöhnlich zu den am wenigsten genutzten futurischen Ausdrucksmitteln (in V-5P_MBeh erreicht die Form mit 3,4\% aller futurischen Belege einen vergleichsweise hohen Anteil während in MMag und GnaÜ nur Einzelbelege vorliegen).

14 4,1\% bei Matzel/Ulvestad (1982: 290f.), 3,3\% bei Saltveit in der geschriebenen Sprache und 10,9\% in der Mundart (vgl. Matzel/Ulvestad, ebd.). 


\section{Zusammenfassung}

Zusammenfassend lässt sich festhalten, dass im untersuchten Korpus bei der Bezeichnung zukunftsbezogener Sachverhalte eine gesamthafte Entwicklung erkennbar ist. Einer allgemeinen Rückläufigkeit der Verwendung des Präsens in entsprechenden Äußerungen steht eine verstärkte Verwendung der Modalverbperiphrasen wil + Inf. und sol + Inf. gegenüber, muoz + Inf. folgt dieser Entwicklung nicht. In den Nebensätzen ist die Veränderung des Formengebrauchs wesentlich weniger deutlich, hier bleibt meist das Präsens vorherrschend. Zu den beiden Konstruktionen mit werden können kaum allgemeine Aussagen getroffen werden, da sie nur selten auftreten, insbesondere werden + Inf. bleibt auch im letzten Untersuchungszeitraum marginal.

Die Einzelbetrachtung der Handschriften zeigt oftmals typische Gebrauchsweisen der Formen, die mit kontextuellen, inhaltlich-thematischen und vermutlich auch stilistischen Aspekten in Zusammenhang stehen. Meist wird in den Hss. eine Modalverbkonstruktion bevorzugt, während die anderen deutlich seltener verwendet werden. Dennoch bleibt das Präsens in der Mehrzahl der Hss. in der gesamten Untersuchungsperiode die bevorzugte Ausdrucksform für zukünftige Inhalte.

Die Ergebnisse zur Formenverteilung sind für das untersuchte Korpus repräsentativ. Die allgemeinen Entwicklungstendenzen - Rückgang des Präsensgebrauchs und Zunahme der Modalverbkonstruktionen - können auch als Merkmal des Ausbaus des analytischen Sprachbaus aufgefasst werden. Es ist anzunehmen, dass die hier ermittelte Grundtendenz auch für das gesamte Bochumer Mittelhochdeutschkorpus gilt. Bei ähnlichen Untersuchungen ist grundsätzlich eine entscheidende Bedeutung der Korpuszusammenstellung zu berücksichtigen. Als kritischer Faktor kommt außerdem die Abhängigkeit des Ergebnisses von der Interpretation der Belege hinzu. Obwohl nur Belege aufgenommen wurden, deren Auswahl sich im Hinblick auf den jeweiligen Text gut begründen lässt, ist eine gewisse Subjektivität auf Seiten des Auswertenden nie gänzlich auszuschalten. 


\section{Literatur}

Abraham, Werner (1989): Futur-Typologie in den germanischen Sprachen, in: Tempus - AspektModus, Die lexikalischen und grammatischen Formen in den germanischen Sprachen, hrsg. v. Werner Abraham u. Theo Janssen, Tübingen: 345-389.

Bech, Fedor (1901): Beispiele von der Abschleifung des deutschen Participium Präsentis und von seinem Ersatz durch den Infinitiv, in: Zeitschrift f. dt. Wortforschung 1, Heft 2: 81-109.

Behaghel, Otto (1924): Deutsche Syntax, Eine geschichtliche Darstellung, Heidelberg.

Bogner, Istvan (1989): Zur Entwicklung der periphrastischen Futurformen im Frühneuhochdeutschen, in: ZfdPh 108: 56-85.

Bogner, Stephan (1994): Periphrastische Futurformen im Frühneuhochdeutschen, Wien.

Brons-Albert, Ruth (1982): Die Bezeichnung von Zukünftigem in der gesprochenen deutschen Standardsprache, Tübingen.

Bybee, Joan L. (1985): Morphology. A study of the relation between meaning and form, Amsterdam.

Bybee, Joan L./Pagliuca, William (1987): The evolution of future meaning, in: Papers from the VIIth International Conference on Historical Linguistics, hrsg. v. A. G. Ramat, O. Carruba and G. Bernini, Amsterdam: 109-122.

Bybee, Joan L./Perkins, Revere D./Pagliuca, William (1994): The evolution of grammar, Tense, aspect, and modality in the languages of the world, Chicago.

Dahl, Östen (1985): Tense and aspect systems, Oxford.

Duden - die Grammatik (2005). 7., völlig neu erarb. und erw. Aufl., hrsg. v. der Dudenredaktion, Mannheim.

Ebert, Robert Peter/Reichmann, Oskar/Solms, Hans-Joachim/Wegera, Klaus-Peter (1993): Frühneuhochdeutsche Grammatik, Sammlung kurzer Grammatiken germanischer Dialekte A, Hauptreihe, Nr. 12, Tübingen.

Eroms, Hans-Werner (1992): Das deutsche Passiv in historischer Sicht, in: Deutsche Syntax. Ansichten und Aussichten, hrsg. v. Ludger Hoffmann. Berlin: 225-249.

Fritz, Thomas (1995): Deontische, epistemische und futurische Verwendungsweisen von Modalverben um 1400, in: Aspekte der Sprachbeschreibung, hrsg. v. Per Bærentzen, Tübingen: 51-54. 
Fritz, Thomas (1997): Zur Grammatikalisierung der zusammengesetzten Verbformen mit werden werden und die Modalverben im frühen Deutsch und heute, in: Zu Tempus und Modus im Deutschen, hrsg. v. Heinz Vater, FOKUS, Linguistisch-Philologische Studien, Bd. 19, Trier: 81-104.

Gelhaus, Hermann (1969): Der Begriff Tempus - eine Ansichtssache?, Düsseldorf(Wirkendes Wort, Beiheft 20).

Herbers, Birgit (2002): Verbale Präfigierung im Mittelhochdeutschen, eine semantisch-funktionale Korpusanalyse, Studien zur Mittelhochdeutschen Grammatik 1, Tübingen.

Klein, Thomas (1991): Zur Frage der Korpusbildung und zur computerunterstützten grammatischen Auswertung mittelhochdeutscher Quellen, in: ZfdPh 110: 3-23.

Klein, Thomas/Solms, Hans-Joachim/Wegera, Klaus-Peter (2009): Mittelhochdeutsche Grammatik, Teil 3 Wortbildung, Tübingen.

Kleiner, Mathilde (1925): Zur Entwickelung der Futur-Umschreibung werden mit dem Infinitiv, Berkeley.

Kotin, Michail (2003): Die werden-Perspektive und die werden-Periphrasen im Deutschen, Historische Entwicklung und Funktionen in der Gegenwartssprache, Frankfurt/Main.

Laswon, Richard H. (1958): The Old High German Translations of Latin Future Active in Tatian, in: JEGP 57: 64-71.

Leipold, Aletta (2006): Verbableitung im Mittelhochdeutschen, Eine synchron-funktionale Analyse der Motivationsbeziehungen suffixaler Verbwortbildungen, Studien zur Mittelhochdeutschen Grammatik 2, Tübingen.

Leiss, Elisabeth (1985): Zur Entstehung des neuhochdeutschen analytischen Futurs, in: Sprachwissenschaft 10: 250-273.

Leiss, Elisabeth (1992): Die Verbalkategorien im Deutschen, Ein Beitrag zur Theorie der sprachlichen Kategorisierung, Studia Linguistica Germanica 31, Berlin.

Lussky, George F. (1924): ,Uuerdan' und ,Uuesan' mit dem Partizip Passiv in der ahd. Tatianübersetzung, in: JEGP 23, 3: 342-369.

Matzel, Klaus/Ulvestad, Bjarne (1982): Futur I und futurisches Präsens, in: Sprachwissenschaft 7: 282-328.

Paul, Hermann (2007): Mittelhochdeutsche Grammatik, 25. Aufl. neu bearbeitet v. Thomas Klein, Hans-Joachim Solms u. Klaus-Peter Wegera, mit einer Syntax von Ingeborg Schröbler, neu bearbeitet u. erweitert v. Heinz-Peter Prell, Sammlung kurzer Grammatiken germanischer Dialekte A, Hauptreihe Nr. 2, Tübingen. 
Pfefferkorn, Oliver (2005): Die periphrastischen Futurformen im Mittelhochdeutschen, in: Sprachwissenschaft 30: 309-330.

Saltveit, Laurits (1957): Einige Bemerkungen zum deutschen Futur, in: ZfDA 87: 213-228.

Saltveit, Laurits (1960): Besitzt die deutsche Sprache ein Futur?, in: Der Deutschunterricht 12: $46-65$.

Saltveit, Laurits (1962): Studien zum deutschen Futur, Bergen/Oslo.

Schieb, Gabriele (1981): Der Verbkomplex aus verbalen Bestandteilen, in: Zur Ausbildung der Norm der deutschen Literatursprache (1470-1730) I, hrsg. v. Gerhard Kettmann u. Joachim Schildt, Berlin: 39-234.

Schmid, Hans Ulrich (2000): Die Ausbildung des werden-Futurs, Überlegungen auf der Grundlage mittelalterlicher Endzeitprophezeiungen, in: Zeitschriftf. Dialektologie und Linguistik 67: 6-27.

Schrodt, Richard (2004): Althochdeutsche Grammatik II, Syntax, Tübingen.

Szatzker, Szilvia (2002): Zur Grammatikalisierung von werden/würde + Inf. im Neuhochdeutschen (1650-2000), Diss. Budapest.

Thieroff, Rolf (1992): Das finite Verb im Deutschen. Tempus - Modus - Distanz, Tübingen.

Thieroff, Rolf (1994): Tense systems in European languages. 2 Bde., Tübingen.

Ultan, Russell (1988): The Nature of Future Tenses, in: Universals of Human Language. Vol. 3 Word Structure, hrsg. v. Joseph H. Greenberg, Stanford: 83-123.

Vater, Heinz (1975): Werden als Modalverb, in: Aspekte der Modalität, hrsg. v. J.P. Calbert u. H. Vater, Tübingen: 71-148.

Vater, Heinz (1997): Hat das Deutsche Futurtempora?, in: Zu Tempus und Modus im Deutschen, hrsg. v. Heinz Vater, Trier: 53-69.

von Monsterberg-Münckenau, Sylvius (1886): Der Infinitiv nach ,wellen' und den Verba Praeteritopraesentia in den Epen Hartmanns von Aue, in: ZfdPh 18: 1-54, 144-185, 301-320.

Waldenberger, Sandra (2009): Präpositionen und Präpositionalphrasen im Mittelhochdeutschen, Studien zur mittelhochdeutschen Grammatik 3, Tübingen.

Walther, Claus (1980): Untersuchungen zu Häufigkeit und Funktionen des deutschen Futurs (werden + Inf.) in hochdeutschen Texten zwischen 1450 und 1750 (mit einem Ausblick ins Niederdeutsche), Diss. Berlin. 
Walther, Claus (1982): Einblicke in die Geschichte unserer Futurform (werden + Inf.), in: Wissenschaftl. Zeitschr. d. Humboldt-Universität zu Berlin, Ges.-Sprachw. R. XXX1: 597-601.

Wegera, Klaus-Peter (2000): Grundlagenprobleme einer mittelhochdeutschen Grammatik, in: Sprachgeschichte. Ein Handbuch zur Geschichte der deutschen Sprache und ihrer Erforschung, Band 2, 2. Aufl. Berlin: 1204-1320.

Weinhold, Karl (1883): Mittelhochdeutsche Grammatik, Breslau (2. Ausg., unveränd. [fotomechan.] Nachdr. 1967, Paderborn).

Welke, Klaus (2005): Tempus im Deutschen, Rekonstruktion eines semantischen Systems, Berlin.

Westvik, Olaf Jansen (2000): Über Herkunft und Geschichte des werden-Futurs. Eine Auseinandersetzung mit neuerer und neuester Forschung, in: Raum, Zeit, Medium - Sprache und ihre Determinanten. Festschrift für Hans Ramge zum 60. Geburtstag, hrsg. v. Gerd Richter u.a., Darmstadt: $235-261$.

Wilmanns, Wilhelm (1906): Deutsche Grammatik, Gotisch, Alt-Mittel- und Neuhochdeutsch, Bd. 3, I, Strassburg.

Winkler, Johanna (1913): Die periphrastische Verbindung der Verba sîn und werden mit participium praesentis im Mittelhochdeutschen des 12. und 13. Jahrhunderts, Diss. Heidelberg.

Žuikin, Juri N. (1975) : Futur I und futurisches Präsens im unabhängigen Satz, in: DaF 12: 44-50. 
Anhang: zitierte Handschriften

Sigle

I-0-P_Will

I-0-P_WNot Wiener Notker

II-2-P_SPec Speculum ecclesiae

II-3-V_LEntc Linzer Entechrist

II-4-V_ArnM Arnsteiner Mariengebet

II-5-P_PrFr Frankfurter Predigtfragmente

III-0-V_Nib Nibelungenlied (C)

III-0-V_Parz Wolfram von Eschenbach: Parzival (D)

III-5-P_PrMK Mitteldeutsche Predigten

IV-3-P_PrSch Schwarzwälder Predigten

IV-4a-P_Lilie Die Lilie (Prosateil)

IV-5-P_AthP Athis und Prophilias

\section{Handschrift}

Breslau, Bibl. uniw., cod. R 347

Wien, ÖNB, Cod. 2681

München, BSB, Cgm 39 [Hs. C], fol. $4 \mathrm{r}-132 \mathrm{v}$ u. $142 \mathrm{r}-178 \mathrm{v}$

Linz, LB, cod. 33, fol. 171r-180r

Wiesbaden, HStA, Hs. Abt. 3004, C 8, fol. $129 \mathrm{v}-135 \mathrm{v}$

Frankfurt, SUB, Fragm. germ. I 1

Karlsruhe, LB, Cod. Donau-eschingen 63 , fol. 1r-89r

St. Gallen, StiB, Cod. 857

Berlin, SBPK, Fragm. 55 [Fragm. $\mathrm{K}]$

Freiburg, UB, Cod. 460 [Gr]

Wiesbaden, HessLB, Hs. 68

Krakau, BJ, Berol. mgq 846

[ABCDEF]; Berlin, SBPK, Nachlaß Grimm 196 (angebunden) $\left[A^{*} C^{*}\right]$ 
V-1-V_MMag Maria Magdalena

V-2-P_Baum Baumgarten geistlicher Herzen (L)

V-3-P_NikP Nikolaus von Straßburg, Predigten (C)

V-4a-P_Taul Johannes Tauler, Predigten

V-5-P_MBeh Evangelienbuch des Matthias von Beheim

V-6-P_GnaÜ Christina Ebner, Von der Gnaden Überlast
Wien, ÖNB, Cod. 15225, suppl. 3146, fol. $1 \mathrm{r}-33 \mathrm{r}$

München, BSB, Cgm 6247 [Hs. L]

St. Florian, StiB, Cod. XI 284, kl. $4^{\circ}$, fol. 37ra, $1-85 \mathrm{rb}, 3$ [Hs. C]

Wien, ÖNB, Cod. 2744

Leipzig, UB, Ms. 34, fol. 53r224r (Evangelienbuch) u. fol. 224v-234v (Harmonie der Passionsgeschichte)

Nürnberg, GNM, Hs. 1338 [N2] 